\section{Bone-targeted agents in multiple myeloma}

\section{Hiroko Nishida}

Department of Pathology, Keio University, School of Medicine, Tokyo, Japan

\begin{abstract}
Osteolytic bone disease, characterized by bone pain, increased risk of pathologic fractures, tumor-induced hypercalcemia known as skeletal-related events (SREs), is a frequent complication of patients with multiple myeloma (MM) and persists even in the absence of active disease, resulting in a major cause of morbidity and mortality. The interaction between myeloma cells and their surrounding cells in the bone marrow (BM) microenvironment promotes both myeloma cell growth and bone destruction and forms the vicious cycle of MM bone disease. Therefore, therapeutic strategies targeting the interaction between myeloma cells and cellular components including osteoclasts (OCs), stromal cells and osteoblasts (OBs) in the BM is crucial not only to attain tumor regression but also to prevent or delay the incidence of SREs, which leads to improve survival and quality of life in affected patients. Recently, several novel targets which act on components of the cycle for treating MM-associated bone disease have been identified in addition to current treatments including nitrogen-containing bisphosphonates. This review focuses on the overview of pathophysiology in MM-associated bone disease and summarizes its current clinical management. Several novel bone-targeted agents in preclinical setting will be also discussed.
\end{abstract}

\section{Introduction}

The bone is a common site of metastasis in patients with advanced cancer such as breast cancer, prostate cancer, lung cancer and multiple myeloma (MM). ${ }^{1-3} \mathrm{MM}$ is a hematological malignancy, characterized by the accumulation of monoclonal plasma cells in the bone marrow (BM) and a recent significant advance in the treatment options of MM has become improved median overall survival (OS) in MM patients. In the BM microenvironment of $\mathrm{MM}$, the cellular interaction promotes myeloma cell growth and devastates its associated bone disease, characterized by pathologic fractures, spinal cord compression or hypercalcemia, known as skeletal related events (SREs). ${ }^{4-9}$ The frequency of SREs depends on the characteristics of bone lesions, locations and treatment complications. Despite improvement in treatment options, many patients with MM experience unsatisfactory pain which severely compromises their quality of life (QOL). Approximately 70\% of patients have bone disease at the initial diagnosis of $\mathrm{MM}$ and $80-90 \%$ of patients develop SREs during its clinical course. , $^{9} 10$ Due to the uncoupled bone remodeling process, MM-associated bone lesions are osteolytic with bone loss. Myeloma cells promote osteoclast (OC) formation in association with BM stromal cells, whereas they inhibit osteoblast (OB) formation, which result in increased bone destruction and suppressed bone formation. BM accessory cells including OCs, BM stromal cells (BMSCs), endothelial cells and immune cells supply a microenvironment suitable for MM cell expansion. ${ }^{11-13}$ Thus, the interaction between MM cells and these cellular components in the BM results in the vicious cycle to expand MM cells and destructive bone lesions (Figure 1). ${ }^{14}$ Importantly, MMassociated bone disease dose not completely heal but persists even in the absence of active disease of MM. Therefore, despite remarkable progress in treatments of MM over the last decade, to develop novel therapeutic strategies targeting osteolytic bone disease is required to attain improved QOL in the long-term management of MM patients. $^{4-9}$ This review focuses on the mechanism of the interaction between MM cells and their surrounding cells in the BM and summarizes current clinical managements in MM- associated bone disease to prevent or delay the occurrence of SREs in affected patients. In addition, several novel targets in preclinical setting or clinical trials will be discussed for providing clues further to develop novel therapeutic agents.

\section{Pathophysiology of multiple myeloma associated bone disease}

The interaction between MM cells and BM microenvironment plays an important role in the pathogenesis of MM. ${ }^{11-14}$ Soluble factors or physical contacts within the cellular components of BM have been implicated in both enhanced bone destruction and impaired bone formation in MM. BM microenvironment is composed of various kinds of cells including BM stromal cells (BMSCs), osteocytes, OCs, osteoblasts (OBs), vascular endothelial cells and immune cells.
Correspondence: Hiroko Nishida, Department of Pathology, Keio University, School or Medicine, 35 Shinanomachi, Shinjuku-ku, 160-8582, Japan.

Tel.: +81.3.5363.3785 - Fax: +81.3.3353.3290

E-mail: hiroko@a2.keio.jp

Key words: skeletal-related events, multiple myeloma, osteoclast, quality of life, bisphosphonate, novel bone-targeted agents.

Funding: This work is supported by Grants-inAid for Scientific Research from the Ministry of Education, Culture, Sports, Science and Technology of Japan (16K07180) to H. Nishida.

Conflict of interest: the author declares no conflict of interests.

Received for publication: 4 September 2017. Revision received: 16 November 2017.

Accepted for publication: 11 December 2017.

This work is licensed under a Creative Commons Attribution-NonCommercial 4.0 International License (CC BY-NC 4.0).

(C) Copyright H. Nishida, 2018

Licensee PAGEPress, Italy

Hematology Reports 2018; 10:7401

doi:10.4081/hr.2018.7401

\section{Osteoclast activation}

MM cells produce factors including receptor activator of $\mathrm{NF}-\kappa \mathrm{B}$ ligand (RANKL), macrophage inflammatory protein-1 $\alpha$ (MIP1- $\alpha$ ), interleukin-3 (IL-3) and interleukin-6 (IL-6), 15-18 in the BM. When MM cells bind to BMSCs, RANKL expression in BMSCs is increased, which results in the binding of RANKL to RANK on OC precursor cells and OC differentiation is induced. Likewise, T-lymphocytes also produce RANKL and IL-3 to enhance osteoclastgenesis. In addition, the interaction between MM cells and BMSCs leads to decreased osteoprotegrin (OPG) produced by BMSCs which inhibits the action of RANKL. Consequently, marked imbalance between RANKL and OPG levels in the BM microenvironment of MM triggers OC activation and $\mathrm{OB}$ suppression. ${ }^{16}$ Indeed, RANKL expression was remarkably up-regulated, whereas OPG levels was down-regulated in the BM microenvironment of MM patients. In a murine model of MM, a RANKL inhibitor, RANK-Fc, was shown to inhibit osteoclastgenesis and bone resorptive activity. ${ }^{19}$ Based on these observations, a human monoclonal $\mathrm{IgG}_{1}$ antibody against RANKL has been developed and discussed as below. Moreover, both MIP1- $\alpha$ and MIP1$\beta$ secreted by MM cells induce both RANKL up-regulation and OPG down-regulation in 
BMSCs through the interaction between VLA-4 and VCAM-1. ${ }^{19}$ MM cells express MIP1 receptors; CCR5 and CCR1. Both MIP1- $\alpha$ and MIP1- $\beta$ act on MM cells in autocrine an paracrine fashion and stimulate osteoclastgenesis. ${ }^{17,20} \mathrm{IL}-3$ level is elevated in $75 \%$ of BM in MM patients and enhances the effect of RANKL and MIP1, which results in OC activation and bone destruction. Interleukin-6 (IL-6) level is also up-regulated in patients with MM-associated osteolytic bone lesions compared with those without bone lesions or with monoclonal gammopathy of undetermined significance (MGUS). It is produced by OCs, BMSCs and $\mathrm{OBs}$ through the interaction with $\mathrm{MM}$ cells and increase in the level of IL- 6 augments MM cell expansion and bone destruction. Furthermore, in addition to RANKL, BMSC secretes various cytokines and growth factors, including vascular endothelial growth factor (VEGF), stromal cellderived factor (SDF)-1 $\alpha$, and insulin-like growth factor (IGF)-1 as well as physical contacts with MM cells for MM cell proliferation. Another osteoclastgenetic factors including tumor necrosis factor $\alpha(\mathrm{TNF} \alpha), \mathrm{B}$ cell activating factor of $\mathrm{TNF} \alpha$ family (BAFF), A proliferation inducing ligand
(APRIL) are also produced and secreted by OCs in bone lesions in MM. ${ }^{21,22}$

\section{Osteoblast suppression}

MM cells secrete several factors to inhibit OB differentiation, including dickkopf-1 (DKK-1), frizzled-related protein (sFRP)-2, sFRP-3, IL-3 and IL-7. Winglesstype (WNT) signaling pathway plays a crucial role in OB differentiation. ${ }^{23-26}$ Complex of WNT and LDL-related receptor (LRP) 5 or LRP6 binds to the fizzled receptor, leading to dephosphorylation of $\beta$-catenin pathway which activates OB differentiation. DKK1 is WNT pathway inhibitor which is up-regulated in MM cells and is more intensely expressed in patients with multiple osteolytic lesions than those without these lesions. Likewise, DKK1 expression is up-regulated in BMSCs and OBs in the presence of MM cells. DKK1 binds to LRP-5 and inhibits the binding of WNT to LRP5, resulting in blockade of downstream signaling pathway and inhibition of osteoblastgenesis. Moreover, DKK1 increases RANKL expression and decrease OPG expression in OBs, enhancing osteoclastgenesis in MM. sFRP-2 and sFRP3, secreted by MM cells are WNT antagonists which inhibits $\mathrm{OB}$ differentiation.
Endogeneous sFRP-2 blocked alkaline phosphatase activity and suppressed mineralized nodule formation enhanced by bone morphogenetic protein (BMP)-2. In MM patients, IL-3 enhances bone destruction by enhancing osteolastgenesis and indirectly inihibits osteoblastgenesis. IL-7 suppressed Runx2 activation which is required for osteoblast precursor differentiation. ${ }^{27,28}$

\section{Bone-targeted agents in multiple myeloma}

MM is an incurable disease with antiMM agents including proteasome inhibitors (PIs), immunomodulatory drugs (IMiDs), immunotherapy and hematopoietic stem cell transplantation. Patients with MM frequently experience pathologic fractures, spinal cord compression or hypercalcemia, known as SREs and experience unsatisfactory pain which significantly compromises their QOL. ${ }^{4-9}$ Current therapeutic options of MM-associated bone disease include nitrogen-containing bisphosphonates, surgical procedures, radiotherapy and systemic antiMM therapy itself.

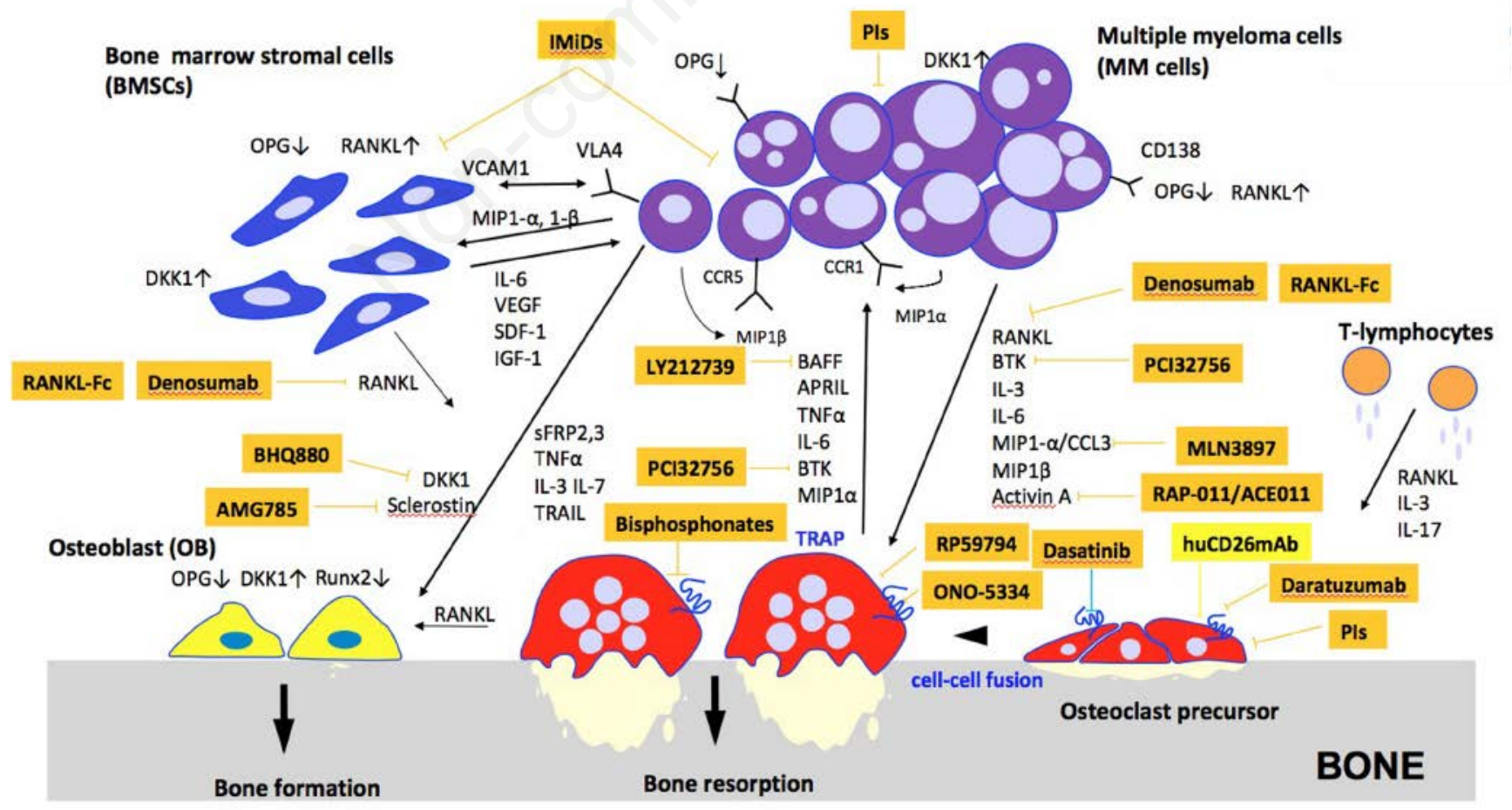

Figure 1. Vicious cycle of multiple myeloma-associated bone disease. 


\section{Bispohsphonates}

Nitrogen-containing bisphosphonates including zoledronate and pamidronate are currently used to control and alleviate bone pain, to prevent the incidence of SREs, to reduce the requirement for palliative radiation to affected bone or surgery, and to delay time to first SREs. ${ }^{4-9}$ The mechanism of action of bisphosphonates is to inhibit protein prenylation and to block prenylate GTPase signaling required for OC formation by suppressing farnesyl pyrophosphate synthetase (FPPS), the enzyme in the mevalonate pathway. ${ }^{29}$ Bisphosphonates maintain high affinity for hydroxyapatite surface on the bone through their similarity to pyrophosphates and are incorporated into bone remodeling sites and released for months, resulting in the inhibition of bone resorption. In addition, bisphosphonates increase apoptosis of MM cells and perform anti-angiogenetic effect by inhibiting the expression of vasoendothelial growth factor (VEGF) or platelet derived growth factor (PDGF) in endothelial cells. In contrast, non-nitrogen containing bisphosphonate such as clodronate induces OC apoptosis not by the induction of protein prenylation but the formation of nonhyrdolyzable ATP metabolites. Nitrogen-containing bisphosphonates have been shown to prolong median OS and progression free survival (PFS) as compared to oral clodronate, in combination with anti-myeloma therapy. ${ }^{30}$ Recent guidelines by International Myeloma Working Group (IMWG) and National Cancer Comprehensive Network (NCCN) recommended the initiation of bisphosphonate therapy for MM patients regardless of the presence or absence of osteolytic bone lesions or osteoporosis. ${ }^{31,32}$ Suggested dose of zoledronate and pamidoronate is $4 \mathrm{mg}$ every 3 to 4 weeks as a 15 minute infusion and $90 \mathrm{mg}$ every 3 to 4 weeks as a 2 hour infusion in symptomatic MM, ${ }^{33}$ whereas dosing recommendation of bisphosphonates for asymptomatic or smoldering $\mathrm{MM}$ is varying. High-risk smoldering MM patients with osteolytic bone lesions should receive bisphosphonate treatment with the same dose and schedule used for symptomatic MM patients. On the other hand, other smoldering or asymptomatic MM patients were annually treated with the dose $(5 \mathrm{mg})$ used for patients with osteoporosis. ${ }^{33}$ Recent reports suggest that zoledronate was shown to be effective in prolonging time to the first SRE in advanced cancer and bone metastasis. ${ }^{34-36}$ However, there were no evident data which demonstrate the superiority of zoledoronate over pamiodronate. Moreover, Rosen et al. demonstrated that zoledronate $(4 \mathrm{mg})$ reduced overall risk of developing skeletal complications and hypercalcemia by an additional 16\% in MM patients $(n=1648)$ with bone lesions and have a similar efficacy with pamidoronate, ${ }^{37,38}$ while Sanfilippo et al. reported that zoledronate induced significant improvement in overall survival of MM and reduced its SREs as compared with pamidronate. ${ }^{39}$ Recommended treatment duration of bisphosphonate therapy varies from 2 months to 2 years for MM patients in complete remission (CR) or very good partial remission (VGPR). ${ }^{40}$ Furthermore, it was reported that bisphosphonate therapy should be re-initiated at the time of disease relapse if osteolytic bone disease exists, regardless of the dose of bisphosphonate patients have previously received. ${ }^{33}$ In the aspect of toxicity profiles, bisphosphonates induce osteonecrosis of the jaw (ONJ), renal dysfunction and atypical femoral fractures (AFFs). ${ }^{41-43}$ Renal dysfunction frequently occurs in patients with MM and zoledronate simultaneously exacerbates the renal impairment. Therefore, in several cases, alternative therapeutic agents are required instead of zoledronate further to reduce the occurrence of SREs without drug toxicities.

\section{Denosumab}

Recently, several clinical trials have demonstrated that anti-RANKL monoclonal antibody, denosumab can significantly reduce SREs associated with osteolytic metastatic cancers. ${ }^{44}$ Denosumab is a fully human monoclonal $\mathrm{IgG}_{1}$ antibody which binds to RANKL with high specificity and inhibits RANKL-RANK signaling in OC precursor cells. ${ }^{44}$ Consequently, it blocks OC differentiation and subsequently suppress OC bone resorptive activity with decreased bone destruction. Several trials have shown that denosumab was superior to zoledronate in delaying or preventing the occurrence of SREs, with median progression free and overall survival similar to zoleronate in osteolytic bone disease including advanced breast cancer, prostate cancer and lung cancer. ${ }^{45-48}$ Although, initial dose adjustment of zoledronate is essential for patients who have baseline creatinine clearance lower than $60 \mathrm{~mL} / \mathrm{min}$, it is not required for denosumab. ${ }^{49}$ Although hypocalcemia is more frequently seen with denosumab than zoledronate, it is manageable with appropriate supplementation with oral calcium and vitamin $\mathrm{D}_{3}{ }^{45-48}$ On the other hand, denosumab increased infections by anti-RANKL effect, so it needs to be careful to use in MM patients. Thus, denosumab has an efficacy with little toxicities, but not be superior to zoledronate in patients with bone metastasis of other advanced cancer or MM. ${ }^{45-47}$ Moreover, it does not significantly decrease tumor burden in MM. ${ }^{48,49}$

\section{Anti-multiple myeloma agents- proteasome inhibitors}

Bortezomib has a potent anti-MM activity. It also impairs osteoclastgenesis via p38MAPK inhibition in early phase and $\mathrm{NF} \kappa \mathrm{B}$ inhibition in later phase. It stimulates mesenchymal stem cell (MSC) differentiation into OBs. It up-regulates the expression of RUNX2 essential for OB formation. The second generation PIs such as carfilzomib and ixazomib also inhibits bone resorptive activity in OCs and promotes osteoblastgenesis. ${ }^{50-52}$

\section{Anti-multiple myeloma agents- immunomodulatory drugs}

IMiDs consists of thalidomide, and its second generation derivatives; lenalidomide and pomalidomide. In addition to their antiMM effects, both lenalidomide and pomalidomide inhibits the activation of PU.1 and pERK, which is transcription factor, crucial for OC precursor differentiation into OCs. Lenalidomide decreases RANKL secretion from BMSCs and normalizes the RANKL/OPG ratio. It also inhibits the cathepsin $\mathrm{K}$, essential for bone matrix degradation, secreted by OCs, resulting in decreased osteoclastgenesis and heals oetolytic bone disease in MM, while it does not affect $\mathrm{OB}$ differentiation. ${ }^{53}$

\section{Anti-multiple myeloma agents- monoclonal antibody}

Daratuzumab is a humanized anti-CD38 $\mathrm{IgG}_{1}$ monoclonal antibody. CD38 is expressed in MM cells and antibody-based therapy by daratuzumab revealed significant anti-tumor activity in relapsed or refractory MM patients, which resulted in significant improvements in OS and PFS of MM patients. CD38 is also expressed in OC precursor cells. So, it is expected that daratuzumab not only inhibits tumor growth but also blocks osteoclast differentiation and suppresses osteolytic bone disease in MM. ${ }^{54,55}$ 


\section{Other novel bone-targeted agents-investigational therapies}

\section{Tyrosine kinase inhibitor}

Dasatinib, a multitargeted tyrosine kinase inhibitor, has proven clinically efficient response in bone in combination with zoledronate for the treatment of metastatic breast cancer to bone. ${ }^{56}$ Dasatinib reduces OC number through its inhibitory effect on tyrosine kinase such as macrophage colony stimulating factor receptor (M-CSF), essential for the differentiation of OC precursor cells. Dasatinib also decreases the expression of RANKL and reduces done resorption effect. In addition, dasatinib has bone reserving effects and low dose dasatinib enhances osteoblastgenesis by src inhibition. ${ }^{57,58}$

\section{Cathepsin K inhibitor: ONO-5334}

Cathepsin K, a member of the papain cysteine protease superfamily is released by OCs, has a critical role in bone resorptive activity in mice and degrades collagen of bone. ${ }^{59} \mathrm{ONO}-5334$ is an orally available cathepsin $\mathrm{K}$ inhibitor and its clinical efficacy for increasing bone mineral density (BMD) has been proven in patients with osteoporosis. ${ }^{60}$

\section{MMP inhibitor: RP59794}

MMPs have been involved in recruitment of OCs and had a critical role in bone resorption. An inhibitor which reduces the activities of MMP-2 and MMP-9, relative to other MMPs, efficiently decreases bone resorptive activity. MMP-9 inhibitor inhibited the expression of genes related to OC maturation and suppressed RANKLinduced OC formation. ${ }^{61}$ MMP inhibitor, RP59794 has been shown to reduce OC migration, resulting in the decrease of bone resorption in OCs. ${ }^{62}$

\section{MIP1- $\alpha$ (CCR1) inhibitor: MLN3897}

MIP1- $\alpha /$ CCL3 is a chemokine secreted by $\mathrm{MM}$ cells and plays an important role in MM cell growth and OC differentiation. The receptor for MIP1- $\alpha$ is expressed on cells within the BM microenvironment such as OCs, OBs and BMSCs as well as MM cells. MIP1 $\alpha$ inhibitor, MLN3897, a specific orally available CCR1 inhibitor, impairs ostsoclastgenesis or the interaction between MM cells and OCs and significantly reduced tumor growth in MM. ${ }^{63}$

\section{B-cell activating factor (BAFF) neu- tralizing antibody: LY2127399}

B-cell activating factor (BAFF), which belongs to the TNF superfamily, is secreted by OCs and BMSCs. It increases the adhesion between $\mathrm{MM}$ cell and OCs and enhances MM cell growth. ${ }^{64}$ Anti-BAFF neutralizing antibody, LY2127399 significantly inhibited MM cell growth and subsequently had inhibitory effect on osteoclastgenesis. ${ }^{65}$

\section{Bruton's tyrosine kinase (BTK) inhibitor, PCI-32765 (Ibrutinib)}

BTK, a non-receptor tyrosine kinase, is expressed in B cells and is involved in B cell function. In addition, it is expressed in both MM cells and OCs. A BTK inhibitor, PCI-32765 blocked BTK and PLC $\gamma 2$ in OCs and blocked osteoclastgenesis. It inhibited MM cell growth in vivo and MMinduced osteolyisis in SCID mice. ${ }^{66}$

\section{Activin A (Decay receptor-neutraliz- ing activin A): RAP-011/ACE011}

Activin-A, which belongs to TGF $\beta$ superfamily, is an inhibitor of WNT signaling pathway and is involved in bone remodeling. It inhibits OB differentiation as well as increases OC differentiation. ${ }^{67}$ Interplay between MM cells and BMSCs promotes the secretion of activin A by BMSCs. Indeed, circulating activin A was increased in MM patients and associated with osteolytic bone disease. ${ }^{29-31} \mathrm{~A}$ soluble activin A receptor, RAP-011 increased bone formation, decreased bone disease and reduced tumor growth in murine model of MM. Phase II clinical trials indicated that soluble decay receptor, ACE-011 decreased bone pain and exhibit anti tumor effects in MM patients. ${ }^{68,69}$

\section{Dickkopf-1 (DKK1) neutralizing antibody; BHQ880}

WNT signaling pathway which plays a crucial role in MM bone disease. DDK1 is a WNT antagonist and is found at high levels in MM patients. As disease progresses, it inhibits oseoblastgenesis in MM patients. DKK neutralizing antibody: BHQ880 increased $\mathrm{OB}$ differentiation and also inhibited MM cell growth by modulating BM microenvironment in MM murine model. Phase II clinical trials of BHQ880 in patients with MM revealed increased bone formation. ${ }^{70,71}$

\section{Anti-sclerostin antibody: AMG785}

Sclerostin binds to LRP5/6 and inhibits WNT signaling pathway, which leads to decreased osteoblastgenesis and increased osteoclastgenesis. It also inhibits BMP signaling and suppresses BMP-mediated OB mineralization. In $\mathrm{MM}$ patients, sclerostin levels were shown to be elevated compared with MGUS patients. ${ }^{72}$ A humainized antisclerostin antibody, AMG785 (romosozumab) revealed a significant anabolic effect in the bone and increase BMD in postmenopausal women. ${ }^{73}$ Clinical studies in MM patients have not been conducted yet.

\section{Humanized anti-CD26 mono- clonal antibody}

CD26, 110KDa transmembrane glycoprotein with dipeptidyl peptidase IV (DPPIV) activity, is intensely expressed on OCs in osteolytic bone lesions in MM. Hunmanized anti-CD26 monoclonal antibody has revealed to regulate human OC differentiation by modulating RANK signaling pathway and inactivating downstream intracellular MKK3/6-p38MAPK and intranuclear mi-Mitf phosphorylation pathway in OC precursor cells, thus blocking early OC differentiation. ${ }^{74}$ Bone-targeted therapy with anti-CD26 antibody is expected to reduce the occurrence of total SREs in combination with systemic antiMM therapy.

\section{RANKL-Fc}

A RANKL inhibitor, RANKL-Fc decreases bone resorption induced by RANKL as well as reduces tumor burden in animal models of MM. ${ }^{75}$ Antagonists to MIP1- $\alpha$ receptor, CCR1 blocked OC formation and inhibited the adhesion between MM cells and BMSCs. ${ }^{63}$

\section{Conclusions}

Persistence of unsatisfactory pain due to osteolytic bone disease is a serious problem in MM patients. Bone-targeted therapy in combination with anti-myeloma therapy is crucial to reduce the incidence of SREs and attain an improved QOL for MM patients. Further elucidation of the molecular mechanism underlying MM-associated bone disease may lead to develop novel bone targeted agents and have a dramatic impact on MM tumor growth and osteolytic bone disease.

\section{References}

1. Roodmann GD. Mechanisms of bone metastasis. New Eng J Med 2004;350:1655-64. 
2. Roodman GD. Pathogenesis of myeloma bone disease. Leukemia 2009;23:435-41.

3. Mundy GR. Mechanism of bone metastasis. Cancer 1997;80:1546-56.

4. Heusschen R, Muller J, Duray E, et al. Molecular mechanisms, current management and next generation therapy in myeloma bone disease. Leuk Lymphoma 2017;2:1-15.

5. O'Donnell EK, Raje NS. Myeloma bone disease: pathogenesis and treatment. Clin Adv Hematol Oncol 2017;15:285-95.

6. Silbermann R, Roodman GD. Myeloma bone disease: pathophysiology and management. J Bone Oncol 2013;2:5969.

7. Walker RE, Lawson MA, Buckle CH, et al. Myeloma bone disease: pathogenesis, current treatments and future targets. Br Med Bull 2014;111:117-38.

8. Raje N, Roodman GD. Advances in the biology and treatment of bone disease in multiple myeloma. Clin Cancer Res 2011;17:1278-86.

9. Yeh HS, Berenson JR. Treatment for myeloma bone disease. Clin Cancer Res 2006;12:6279-84.

10. Melton LJ, kyle RA, Achenbach SJ, et al. Fracture risk with multiple myeloma: a population-based study. J Bone Miner Res 2005;20:487-93.

11. Roodman GD. Targeting the bone microenvironment in multiple myeloma. J Bone Miner Metab 2010;28:24450.

12. Hideshima T, Mitsiades C, Tonon G, et al. Undestanding multiple myeloma pathogenesis in the bone marrow to identify new therapeutic targets. Nat Rev Cancer 2007; 7:585-98.

13. Mondello P, Cuzzocrea S, Navarra M. Bone marrow micro-environment is a crucial player for myelomagenesis and disease progression. Oncotarget 2017;8:20394-409.

14. Abe M, Hira K, Wilde J, et al. Osteoclasts enhance myeloma cell growth and survival via cell-cell contact: a vicious cycle between bone destruction and myeloma expansion. Blood 2004;104:2484-91.

15. Wada T, Nakashima T, Hiroshi N, et al. RANKL-RANK signaling in osteoclastgenesis and bone disease. Trends Mol Med 2006;12:17-25.

16. Sezer O, Heider U, Zavrski I, et al. RANK ligand and osteoprotegrin in multiple myeloma bone disease. Blood 2004;101:2094-8.

17. Choi SJ, Cruz JC, Craig F, et al. Macrophage inflammatory protein 1alpha is a potential osteoclast stimulato- ry factor in multiple myeloma. Blood 2000;96:671-5.

18. Lee JW, Chung HY, Ehrlich LA, et al. IL-3 expression by myeloma cells increases both osteoclast formation and growth of myeloma cells. Blood 2004;103:3148-57.

19. Epstein J, Yaccoby S. The SCID-hu myeloma model. Methods. Mol Med 2005;113:183-90.

20. Abe M, Hiura K, Ozaki S, et al. Vicious cycle between myeloma cell binding to bone marrow stromal cells via VLA4VCAM1 adhesion and macrophage inflammatory protein-1 alpha and MIP1 beta production. J Bone Miner Metab 2009;27:16-23.

21. Moreaux J, Legoufffe E, Jourdan E. BAFF and APRIL protect myeloma cells from apoptosis induced by interleukin 6 deprivation and dexamethasone. Blood 2004;103:3148-57.

22. Tai YT, Li X, Breitkreutz I, et al. Role of B-cell activating factor in adhesion and growth of human myeloma cells in the bone marrow microenvironment. Cancer Res 2006;66:6675-82.

23. Gunn WG, Conley A, Deininger L, et al. A crosstalk between myeloma cells and marrow stromal cells stimulates production of DKK1 and interleukin-6: a potential role in the development of lytic bone disease and tumor progression in multiple myeloma. Stem Cells 2006;24:986-91.

24. Tian E, Zhan F, Walker R, et al. The role of the Wnt-signaling antagonists against DDK1 in the development of osteolytic lesions in multiple myeloma. Leuk Lymphoma 2007;48:2323-9.

25. Health DJ, Chantry AD, Buckle CH, et al. Inhibiting dickkopf-1 (DKK1) removes suppression of bone formation and prevents the development of osetolytic bone disease in multiple myeloma. J Bone Miner Res 2009;24:425-36.

26. Fulcininti M, Tassone P, Hideshima T, et al. Anti-DKK1 mab (BHQ) as a potential therapeutic target for multiple myeloma. Blood 2009;14:371-9.

27. Ehrlich LA, Chung HY, Ghobrial I, et al. IL-3 is a potential inhibitor of osteoblast differentiation in multiple myeloma. Blood 2005;106:1407-14.

28. Weitzmann MN, Roggia C, Toraldo G, et al. Increased production of IL-7 uncouples bone formation from bone resorption during estrogen deficiency. J Clin Invest 2002;110:1643-50.

29. Russell RG, Xia Z, Dunford JE, et al. Bisphonates: An update on mechanisms of action and how these relate to clinical efficacy. Ann NY Acad Sci 2007;1117:209-57.
30. Morgab GJ, Child JA, Gregory WM, et al. Effects of zoledronic acid versus clodronic acid on skeletal morbidity in patients with newly diagnosed multiple myeloma (MRC Myeloma IX): secondary outcomes from a randomized controlled trial. Lancet Oncol 2011;12:74352.

31. Anderson KC, Alsina M, Atanackovic D, et al. Multiple myeloma, version 2. 2016: clinical practice guidelines in oncology. J Natl Compr Canc Netw 2015;13:1398-435.

32. Terpos E, Morgan G, Dimopoulod MA, et al. International Myeloma Working Group recommendations for the treatment of multiple myeloma-related bone disease. J Clin Oncol 2013;31:2345-57.

33. Terpos E, Roodman GD, Dimopoulos MA. Optimal use of bisphosphomates in patients with multiple myeloma. Blood 2013;121:3325-8.

34. Rosen LS, Gordon D, Tchekmedyian NS, et al. Long-term efficacy and safety of zoledronic acid in the treatment of skeletal metastases in patients with non small cell lung cancer and other solid tumors: A randomized, Phase III, double-blind, placebo-controlled trial. Cancer 2004;100:2613-21.

35. Saad F, Gleason DM, Murray R, et al. A randomized, placebo-controlled trial o zoledronic acid in patients with hormone-refractory metastatic prostate carcinoma. J Natl Cancer Inst 2002;94:1458-68.

36. Kohno N, Aoki K, Minami H, et al. Zoredronic acid significantly reduces skeletal complications compared with placebo in Japanese woman with bone metastases from breast cancer: A randomized, placebo-controlled trial. J Clin Oncol 2005;23:3314-21.

37. Rosen LS, Gordon D, Kaminski M, et al. Zoledronic acid versus pamidronate in the treatment of skeletal metastases in patients with breast cancer or osteolytic lesion of multiple myeloma. A phase III, double-blind, comparative trial. Cancer J 2001;7:377-87.

38. Rosen LS, Gordon D, Kaminski M, et al. Long-term efficacy and safety of zoledronic acid compared with pamidronate disodium in the treatment of skeletal complications with advanced multiple myeloma or breast carcinoma: A randomized, double-blind multicenter, comparative trial. Cancer 2003; 98:1735-44.

39. Sanfilippo KM, Gage B, Luo S, et al. Comparative effectiveness on survival of zoledronic acid versus pamidronate in multiple myeloma. Leuk Lymphoma 2015;65:615-21. 
40. Morgan GJ, Davies FE, Gregory WM, et al. Effects of induction and maintenance plus long-term bisphosphonates on bone disease in patients with multiple myeloma. Blood 2012:119:5374-83.

41. Walter C, Al-Nawas B, Frickhofen N, et al. Prevalence of bisphosphonates associated osteonecrosis of the jaws in multiple myeloma patients. Head Face Med 2010;6:11

42. Chang JT, Green L, Beitz J, et al. Renal failure with the use of zoredronic acid. N Eng J Med 2003;349:1676-9.

43. Chang ST, Tenforde AS, Grimsrud CD, et al. Atypical femur fractures among breast cancer and multiple myeloma patients recieving intraveneous bisphosphonate therapy. Bone 2012;51:524-7.

44. Castellano D, Sepulveda JM, GarciaEscobar I, et al. The role of RANKLligand inhibition in cancer: the story of denosumab. Oncologist 2011;16:13645.

45. Stopeck AT, Lipton A, Body JJ, et al. Denosumab compared with zoledronic acid or the treatment of bone metastasis in patients with advanced breast cancer: a randomized, double-blind study. J Clin Oncol 2010;28:5132-9.

46. Irelli A, Cocciolone V, Cannita $\mathrm{K}$ et al. Bone targeted therapy for preventing skeletal-related events in metastatic breast cancer. Bone 2016;87:169-75.

47. Fizazi K, Carducci M, Smith M, et al. Denosumab versus zoledronic acid for the treatment of bone metastases in men with castration-resistant prostate cancer: a randomized, double-blind study. Lancet 2011;377:813-22.

48. Body JJ, Facon T, Coleman RE, et al. A study of the biological receptor activator of nuclear factor-kappaB ligand inhibitor, denosumab, in patients with multiple myeloma or bone metastases from breast cancer. Clin Cancer Res 2006;12:1221-8.

49. Henry DH, Costa L, Goldwasser F, et al. Randomized, double-blind study of denosumab versus zoledronic acid in the treatment of bone metastasis in patients with advanced cancer (excluding breast and prostate cancer) or multiple myeloma. J Clin Oncol 2011;29: 1125-32.

50. Zangari M, Esseltine D, Lee CK, et al. Response to bortezomib is associated to osteoblastic activation in patients with multiple myeloma. $\mathrm{Br} \mathrm{J}$ Haematol 2015;131:71-3.

51. Giuliani N, Morandi F, Tagliaferri S, et al. The proteasome inhibitor bortezomib affects osteoblast differentiation in vitro and in vivo in multiple myeloma patients. Blood 2007;110: 334-8.
52. von Metzler I, Krebbel H, Hecht M, et al. Bortezomib inhibits human osteoclastgenesis. Leukemia 2007;21:202534.

53. Breitkreutz I, Raab MS, Vallet S, et al. Lenalidomide inhibits oeteoclastgenesis, survival factors and bone-remodeling markers in multiple myeloma. Leukemia 2008;22:1925-32.

54. Laubach JP, Richardon PG. CD38-targeted immunochemotherapy in refractory multiple myeloma: a new horizon. Clin Cancer Res 2015;21:2660-2.

55. Lokhorst HM, Plesner T, Laubach JP, et al. Tageting CD38 with daratuzumab monotherapy in multiple myeloma. New Eng J Med 2015;373:1207-19.

56. Mitri Z, Nanda R, Blackwell K, et al. TBCRC-010: Phase I/II study of dasatinib in combination with zoledronic acid for the treatment of breast cancer bone metastasis. Clin Cancer Res 2016;22:5706-12.

57. Vandyke K, Dewar AL, Diamond P. The tyrosine kinase inhibitor dasatinib dysregulates bone remodeling through inhibition of osteoclast in vivo. J Bone Miner Res 2010;25:1759-70.

58. Lee Y. Src family kinase/abl inhibitor dasatinib suppresses proliferation and enhances differentiation of osteoblasts. Oncogene 2010;29:3196-207.

59. Tezuka K, Tezuka Y, Maejima A, et al. Molecular cloning of a possible cysteine proteinase predominantly expressed in osteoclasts. J Biol Chem 1994;269:116-9.

60. Tanaka M, Hashimoto Y, Hasegawa C, et al. Antiresponsive effect of a cathepsin K inhibitor ONO-5334 and its relationship to BMD increase in a phase II traial for postmenopausal osteoporosis. BMC Musculskelet Disord 2017;19:267.

61. Kelly T, Borset M, Abe E, et al. Matrix metalloproteinases in multiple myeloma. Leuk Lymphoma 2000;37:273-81.

62. Blavier L, Delasisse JM. Matrix metalloproteinases are obligatory for the migration of preosteoclasts to the developing marrow cavity of primitive long bones. J Cell Sci 1995;108:3649-59.

63. Vallet S, Raje N, Ishitsuka K, et al. MLN3897, a novel CCR1 inhibitor, impairs osteoclastgenesis and inhibits the interaction of multiple myeloma cells and osteoclasts. Blood 2007:110;3744-52.

64. Neri P, Kumar S, Fulciniti MT, et al. Neutralizing B-cell activating factor antibody improves survival and inhibits osteoclastgenesis in a severe combined immunodeficient human multiple myeloma model. Clin Cancer Res
2007;13:5903-9.

65. Raje N, Faber EA, Richardoson PG, et al. Phase 1 study of Tabalumab a human anti-BAFF antibody and bortezomib in patients with previously-treated multiple myeloma. Blood 2012:120;447.

66. Tai YT, Chang AY, Kong SY, et al. Burton tyrosine kinase inhibition is a novel therapeutic strategy targeting tumor in the bone marrow microenvironment in multiple myeloma. Blood 2012;120:1877-87.

67. Takeuchi K, Abe M, Hiasa M, et al. TGF-beta inhibition restores terminal osteoblast differentiation to suppress myeloma growth. Plos One 2010; 5:e9870.

68. Vallet S, Mukherjee S, Vaghela N, et al. Activin A promotes multiple myelomainduced osteolysis and is a promising target for myeloma bone disease. Proc Natl Acad Sci USA 2010;107:5124-9.

69. Abodulkadyrov KM, Salogub GN, Salpgub GN, et al. Sotatercept in patients with osteolytic lesions of multiple myeloma. Br J Haematol 2014;165: 814-23.

70. Heath DJ, Chantry AD, Buckle CH, et al. Inhibiting dickkopf-1 (Dkk1) removes suppression of bone formation and prevents the development of osteolytic bone disease in multiple myeloma. J Bone Miner Res 2009;24:425-36.

71. Fulcininti M, Tassone P, Hideshima T, et al. Anti-DKK1 mab (BHQ) as apotential therapeutic agent for multiple myeloma. Blood 2009;14:371-9.

72. Terpos E, Christoulas D, Katodritou E, et al. Elevated circulating sclerostin correlates with advanced disease features and abnormal bone remodeling in symptomatic myeloma: Reduction postbortezomib monotherapy. Int $\mathrm{J}$ Cancer 2013;131:1466-71.

73. McClung MR, Grauer A, Boonen S, et al. Romosozumab in postmenopausal woman with low bone mineral density. New Eng J Med 2014;370:412-20.

74. Nishida H, Suzuki H, Madokoro H, et al. Blockade of CD26 signaling inhibits human osteoclast development. J Bone Miner Res 2014;29:2439-55.

75. Croucher PI, Shipman CM, Lippitt J, et al. Osteoprotegrin inhibits the development of osteolytic bone disease in multiple myeloma. Blood 2001;98:353440. 